2008

\title{
Helical Axes of Skeletal Knee Joint Motion During Running
}

Antonie J. van den Bogert

Cleveland State University, a.vandenbogert@csuohio.edu

C. Reinschmidt

University of Calgary

A. Lundberg

Huddinge University Hospital

Follow this and additional works at: https://engagedscholarship.csuohio.edu/enme_facpub

Part of the Biomechanical Engineering Commons

How does access to this work benefit you? Let us know!

Publisher's Statement

NOTICE: this is the author's version of a work that was accepted for publication in Journal of Biomechanics. Changes resulting from the publishing process, such as peer review, editing, corrections, structural formatting, and other quality control mechanisms may not be reflected in this document. Changes may have been made to this work since it was submitted for publication. A definitive version was subsequently published in Journal of Biomechanics, 41, 8, (01-01-2008); 10.1016/j.jbiomech.2008.03.018

\section{Original Citation}

van den Bogert, A., Reinschmidt, C., and Lundberg, A., 2008, "Helical Axes of Skeletal Knee Joint Motion during Running," Journal of Biomechanics, 41(8) pp. 1632-1638.

This Article is brought to you for free and open access by the Mechanical Engineering Department at EngagedScholarship@CSU. It has been accepted for inclusion in Mechanical Engineering Faculty Publications by an authorized administrator of EngagedScholarship@CSU. For more information, please contact library.es@csuohio.edu. 


\title{
Helical axes of skeletal knee joint motion during running
}

\author{
A.J. van den Bogert ${ }^{\mathrm{a}, \mathrm{b}, *}$, C. Reinschmidt ${ }^{\mathrm{b}, \mathrm{c}}$, A. Lundberg ${ }^{\mathrm{d}}$ \\ ${ }^{3}$ Department of Biomedical Engineering and Orthopaedic Research Center, The Cleveland Clinic Foundation, 9500 Euclid Avemue, Cleveland, OH 44195, USA \\ ${ }^{\mathrm{b}}$ Faculty of Kinesiology, The University of Calgary, Calgary, Canada \\ ${ }^{\mathrm{c}}$ Zimmer/Centerpulse Orthopedics Limited, Winterthur, Switzerland \\ ${ }^{\mathrm{d}}$ Department of Orthopaedics, Karolinska Institute, Huddinge University Hospital, Stockholm, Sweden
}

\section{Introduction}

Joints are an essential component of musculoskeletal function. The ability of muscles to produce controlled skeletal movements depends on the fact that joints allow substantial amounts of rotation between bones with minimal translation. Joints such as the elbow and the hip consist of highly congruous articular surfaces where this kinematic function is a direct consequence of geometry. Other joints, such as the knee and shoulder, rely on ligaments and muscles to guide the kinematics such that rotation is achieved without undesirable translation. When

\footnotetext{
*Corresponding author at: Department of Biomedical Engineering and Orthopaedic Research Center, The Cleveland Clinic Foundation, 9500 Euclid Avenue, Cleveland, OH 44195, USA. Tel.: + 12164445566 ; fax: +12164449198 .

E-mail address: bogerta@ ccf.org (A.J. van den Bogert).
}

this mechanism fails to function normally, overuse injuries and degenerative joint disease can occur.

An essential functional property of a joint is the axis of rotation, which is defined as the set of points on one bone that have no relative velocity relative to the other bone, at a given time. In analysis of human movement, the intersegmental force and moment vectors are functionally meaningful if they are defined at a "joint center" that lies on this axis of rotation. Since there is no relative velocity at this point, the intersegmental force does no net mechanical work and the function of muscles can be entirely represented by joint moments. In joints with high congruency, a suitable joint center can be defined from geometry (Bell et al., 1990), but this is not possible for the knee joint where the kinematics is only partially governed by the geometry of the articular surfaces. In gait analysis, the knee joint center is typically defined to be the midpoint between the femoral epicondyles. Recently, an average 
knee joint center based on kinematic analysis has been found to be more reproducible than one based on manual palpation (Besier et al., 2003). A fixed axis or center of rotation is, however, inconsistent with the observation of rollback of the femur relative to the tibial plateau (Hill et al., 2000). This implies that the axis of rotation moves posteriorly during flexion, as in a four-bar linkage with inextensible cruciate ligaments (Huson, 1974). Theoretical musculoskeletal models have incorporated such a flexiondependent posterior translation (Delp et al., 1990) so that muscle function can be predicted from musculoskeletal geometry and represented by a moment arm with respect to a functional axis of rotation.

The axis of rotation of the knee joint depends on loading as well as on flexion angle. In vitro studies have shown that at any flexion angle, flexion can occur about different axes, depending on whether the joint is loaded in internal or external rotation (Blankevoort et al., 1990). In addition to this rotational laxity, there is considerable anterior-posterior laxity which indicates that ligaments do not function as inextensible links (Fleming et al., 2002). These observations suggest that external forces and muscles can have a substantial influence on the axis of rotation of the knee joint in vivo. If this is the case, a model with a flexiondependent axis of rotation (Delp et al., 1990) would not correctly represent the functional role of muscles, i.e. moment arms, during movement.

Joint loading, which may alter the axis of rotation, can be incorporated in cadaver experiments (Blaha et al., 2003), but realistic simulations of in vivo loading during functional movement are hard to achieve. The alternative is to study knee joint kinematics in vivo. Medical imaging techniques are typically used to determine in vivo bone motion without skin marker artifacts. Magnetic resonance imaging can be applied during quasi-static conditions (Iwaki et al., 2000; Hill et al., 2000). For dynamic movements, fluoroscopy has proved successful to study in vivo kinematics after total knee replacement, by taking advantage of the high contrast between implant and background (e.g. Dennis et al., 2001; Fantozzi et al., 2003). In natural knee joints, similar information has been obtained through implantation of metallic beads (Jonsson and Karrholm, 1994) or more recently through videofluoroscopy and CT-based bone models (Komistek et al., 2003). Results of these in vivo studies have typically been presented as contact patterns or condylar translations, rather than functional rotation axes or finite helical axes (FHA). This may be due to the high sensitivity of FHA calculations to errors in bone motion data, especially when small rotation steps are used (Woltring et al., 1985). For functional activities such as running, with small rotations during the stance phase, the accuracy, sampling rate and measurement volume requirements for FHA analysis exceed the capabilities of standard medical imaging techniques. Running is of interest because it is associated with large muscle forces acting across the knee joint during stance, which can influence the axis of rotation and play a role in the aetiology of chronic injuries. In a previous study, we have obtained data on 3-D knee kinematics during running, using markers mounted on bone pins, but only rotational motion was reported (Reinschmidt et al., 1997b).

The purpose of the present study was, therefore (1), to describe the FHA of the knee joint during the stance phase of running, and (2) to determine to what extent the observed changes in FHA were dependent on flexion angle.

\section{Methods}

The methodology used to measure the skeletal tibiofemoral kinematics during the stance phase of running is described in detail elsewhere (Reinschmidt et al., 1997a, b). Briefly, intracortical bone pins (Fig. 1) were inserted into the femur and tibia of the right leg of three healthy male subjects (age $25.7 \pm 2.1 \mathrm{yr}$., mass $85.5 \pm 9.6 \mathrm{~kg}$, height $186.7 \pm 9.6 \mathrm{~cm}$ ). The test protocols were approved by the ethics committees of the University of Calgary and the Karolinska Institute, and informed consent was obtained from each subject. Each subject performed five running trials at $2.9 \pm 0.2 \mathrm{~m} / \mathrm{s}$, while the movement of marker triads attached to these pins were recorded with three high-speed cine cameras (LOCAM) recording at $200 \mathrm{~Hz}$. The camera coordinates were low-pass filtered with a double second-order Butterworth filter with a cut-off frequency of $8 \mathrm{~Hz}$. A direct linear transformation (DLT) approach was used to calibrate the cameras and obtain 3-D marker coordinates. Either prior to or after the motion trials, stereo X-rays (frontal and lateral view) were taken with the knee positioned inside a calibration cage for radiostereometric analysis (RSA), again using DLT.

An anatomical tibial coordinate system (ATCS) was defined so that axes of rotation could be reported as a position and orientation relative to

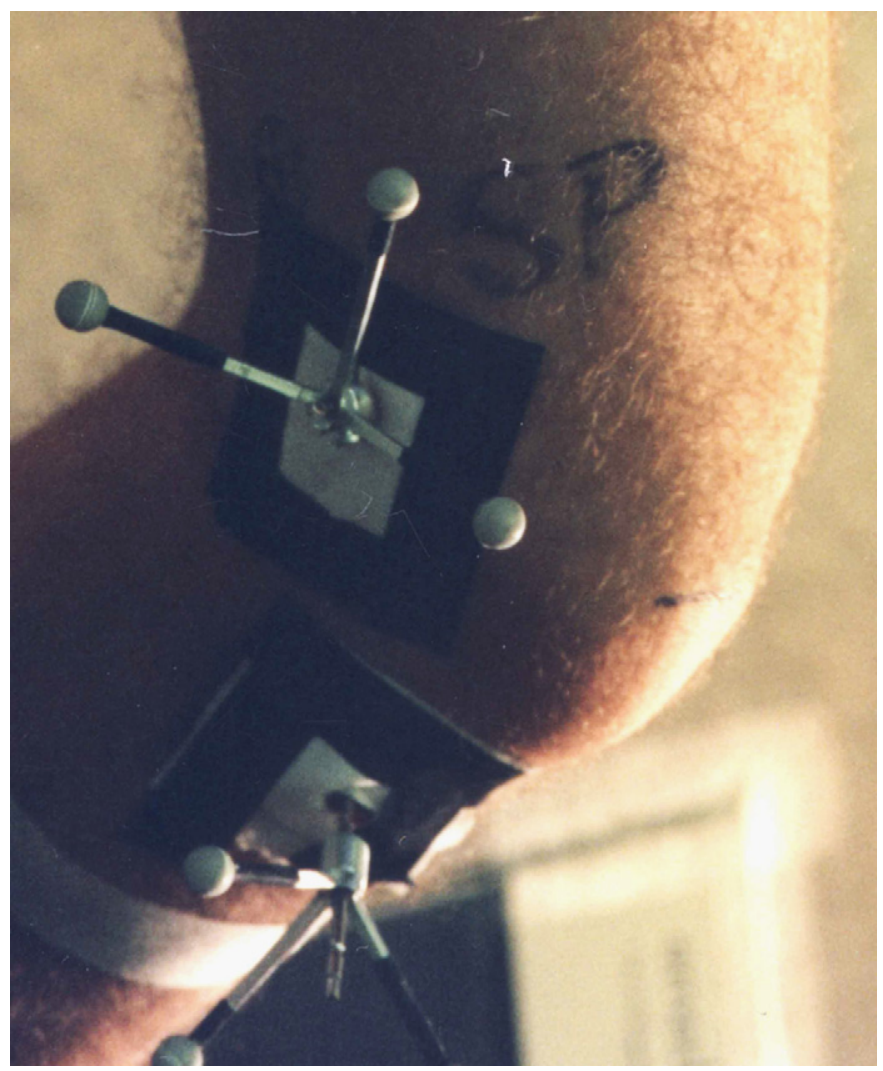

Fig. 1. . Marker triads mounted on intracortical pins located at the lateral epicondyle of the femur and the proximal tibia. 
this reference frame. The 3-D coordinates of the required anatomical landmarks were obtained by manual digitization of the X-rays, as described in detail by Lafortune et al. (1992). Briefly, the most proximal point on the medial intercondylar eminence was chosen as the origin of the ATCS. The direction of the proximal-distal axis ( $y$-axis, proximal positive) was determined by identifying two points on the tibial long axis in both stereo X-ray images. The medio-lateral direction $(z$-axis, lateral positive) was obtained by projecting a line connecting the estimated centers of the medial and lateral tibial articulating surfaces into the plane perpendicular to the proximal-distal axis. The anterior-posterior axis $(x-$ axis, anterior positive) was defined as the cross product of these two axes. The pin-mounted markers were also digitized and their 3-D coordinates were transformed to the ATCS.

KineMat (Reinschmidt and van den Bogert, 1997), a MATLAB ${ }^{\text {TM }}$ (MathWorks, Natick, MA)-based software package, was used for all kinematic analyses. Cardan angles using the conventions of Grood and Suntay (1983) were initially calculated to determine the (time points of) flexion steps for which the helical axes were calculated. The flexion/ extension curves of the three subjects showed a flexion phase during $0 \%$ to approximately $40 \%$ of stance, followed by an extension movement towards the end of stance. For each trial, the flexion phase was divided into four equal steps and the same step size was then used for the extension part of the curve. This resulted into steps of $5^{\circ}, 7^{\circ}$, and $6^{\circ}$ for subject A, B, and C, respectively (Fig. 2).

A singular value decomposition method (Söderkvist and Wedin, 1995) was employed throughout to obtain $4 \times 4$ homogeneous transformation matrices $\mathbf{T}$ from 3-D marker coordinates $r$ measured with respect to different reference frames or at different times:

$r_{\mathrm{A}}=\mathbf{F}_{\mathrm{B} \rightarrow \mathrm{A}} r_{\mathrm{B}}$,

where $r=(x, y, z, 1)^{\mathrm{T}}$ and the subscript A or B indicates the reference frame or time at which the coordinates were measured. For each step (between time 1 and time 2), five sets of marker coordinates were used to calculate the orientation of the helical axes in the RSA coordinate system: $r_{\text {fem } 1}$ (femoral marker coordinates at time 1 ), $r_{\text {fem2 }}$ (femoral marker coordinates at time 2), $r_{\text {tib1 }}$ (tibial marker coordinates at time 1), $r_{\text {tib2 }}$ (tibial marker coordinates at time 2), $r_{\text {tibA }}$ (tibial marker coordinates in the ATCS). The movement of the femur relative to the ATCS between time steps 1 and 2 was described by the following transformation matrix:

$\mathbf{R}=\boldsymbol{\Psi}_{\text {tibl } \rightarrow \text { tibA }} \mathbf{T}_{\text {fem2 } \rightarrow \text { fem } 1} \mathbf{T}_{\text {tibl } \rightarrow \text { tib2 } 2} \mathbf{T}_{\text {tibA } \rightarrow \text { tib1 }}$.

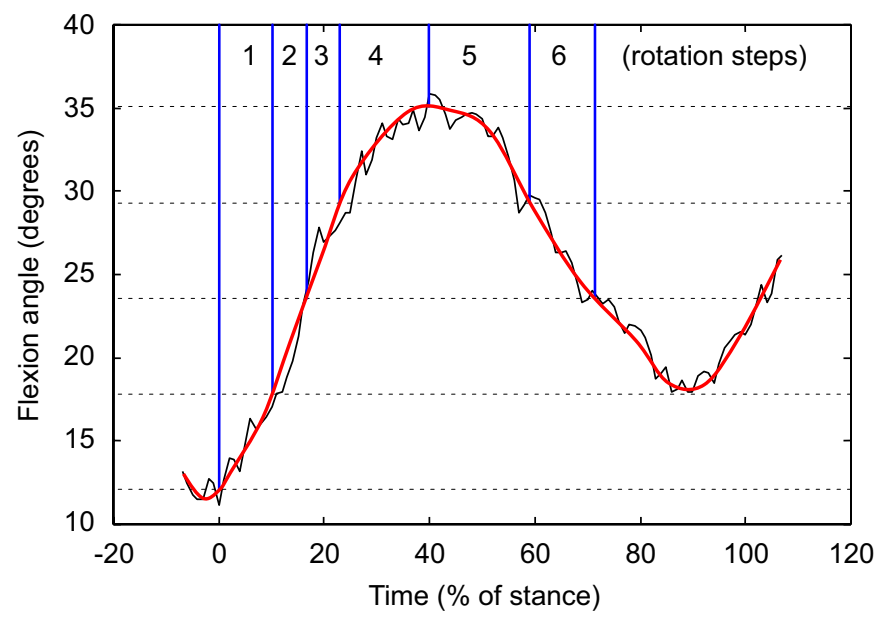

Fig. 2. . Example of flexion angle in one trial, before and after smoothing, showing the six motion steps that were considered in this trial. The flexion phase, starting from heel strike, was divided into four steps with equal amounts of flexion. The number of steps in the extension phase (two, as in this example, or three, or four) depended on the maximal extension achieved during push-off.
The orientation and position of the FHA describing the femur motion between times 1 and 2, with respect to the ATCS, was extracted from $\mathbf{R}$ using the method of Spoor and Veldpaus (1980). The helical axes were projected onto bone tracings in the two X-ray views by using the DLT coefficients of the RSA calibration. For illustrative purposes, medial and a lateral parasagittal planes $\left(160 \times 140 \mathrm{~mm}^{2}\right)$ of the ATCS were also projected onto the X-ray images. The femoral outlines were drawn for the (extended) position in which the RSA images were taken.

The positions and orientations of the FHA were quantified in relation to the ATCS. The deviation (angle between medio-lateral axis of the tibia and the helical axis projected onto transverse tibial plane) and inclination (angle between helical axis and its projection into the transverse tibial plane) were calculated for each flexion/extension step. The positions of the helical axes were also calculated as the intersection of the FHA with the mid-sagittal plane of the ATCS.

The amount of error in the 3-D marker coordinates during running was estimated from the SD of intermarker distances of markers attached to the same intracortical pin (coordinate error $=\mathcal{S D}_{\text {distance }} / \sqrt{ } 2$ ). The error propagation to the final results (orientation and location of the helical axes) was estimated using a Monte Carlo simulation. One trial was arbitrarily selected where the coordinates of all markers were perturbed with normally distributed random errors with known variance to represent the coordinate errors. The perturbation was repeated 1000 times and the RMS (root mean square) differences to the unperturbed axis orientations and positions were calculated.

The error in digitizing the calibration points, bone pin markers and anatomical landmarks on the X-ray images was assessed by repeatedly digitizing these points by two different operators and analysis systems. Note that the anatomical landmarks were first identified by one operator and then marked with a felt pen on the original X-ray image. Therefore, this analysis did not include the reproducibility in defining the anatomical landmarks on the RSA images.

\section{Results}

The coordinate noise for the movement analysis was estimated to be $0.22 \mathrm{~mm}$. Using the Monte Carlo simulation, it was found that this coordinate noise would cause RMS errors of $3.1^{\circ}$ in the deviation and $2.4^{\circ}$ in the inclination of the helical axes. The RMS error in determining the intersection point with the tibial sagittal bisector was found to be $7.6 \mathrm{~mm}$ in the anterior-posterior direction and $6.0 \mathrm{~mm}$ in the proximal-distal direction. The RSA digitization process had errors of less than $0.5 \mathrm{~mm}$, which implies an orientation error for the ATCS of maximally $0.6^{\circ}$.

The helical axis during running stance, when displayed relative to the subject's knee X-rays, were found to be located within the distal femur and have mainly a mediolateral orientation. One typical trial for each subject is shown in Fig. 3.

The orientation and position of the FHA with respect to the ATCS were generally consistent within subjects and between subjects (Fig. 4). In all subjects, the FHAs shifted posteriorly by about $30 \mathrm{~mm}$ during steps $1-5$ and anteriorly from step 6 until the end of extension (Fig. 4A). The proximal-distal position of the FHA was consistently $10-20 \mathrm{~mm}$ above the tibial plateau during the first three flexion steps, followed by a large proximal shift of which the timing was inconsistent between subjects (Fig. 4B). The orientation of the FHA remained within $10^{\circ}$ of the tibial coronal plane (Fig. 4C) but the inclination showed 

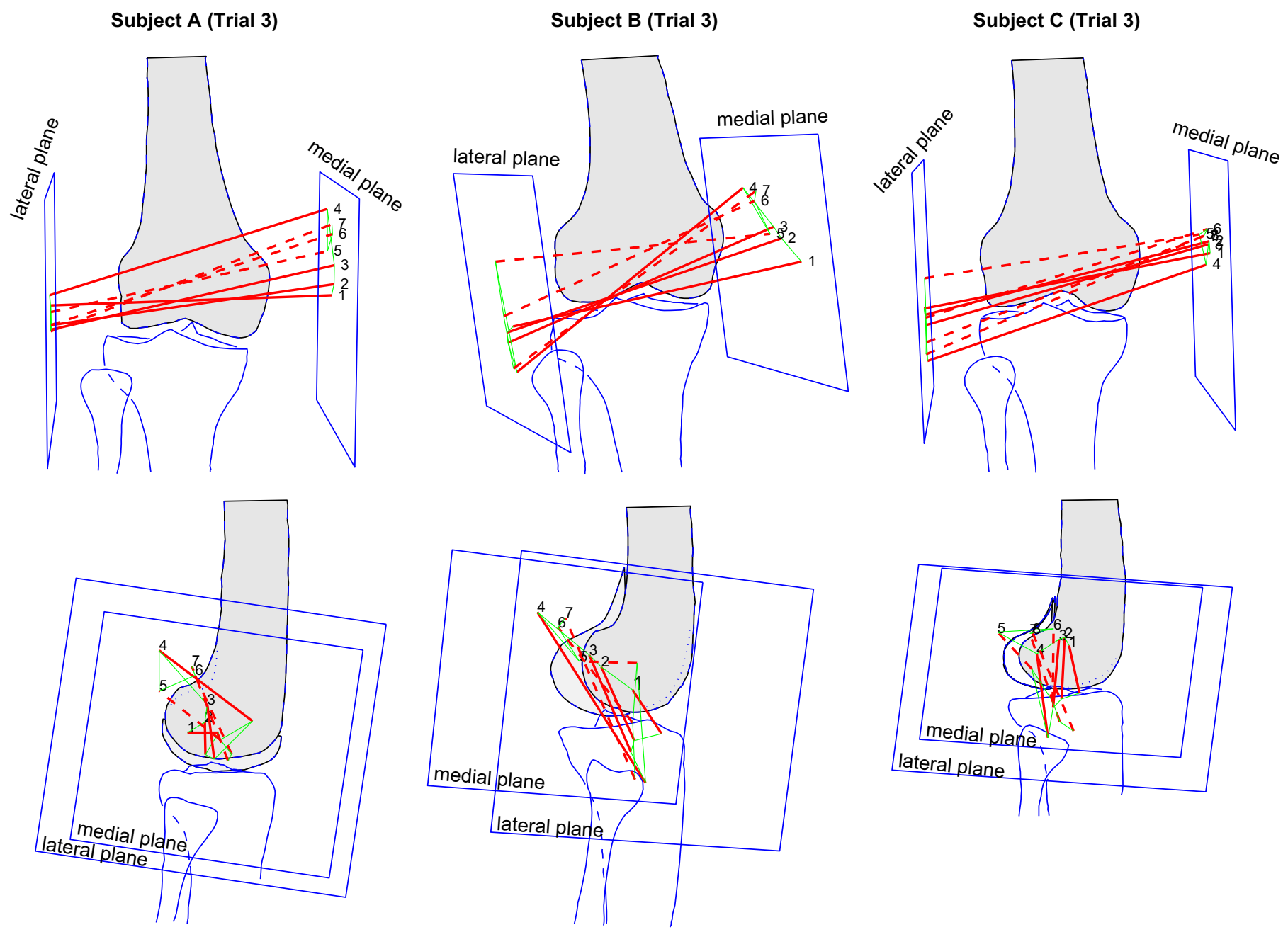

Fig. 3. . Finite helical axes (FHA) projected onto frontal (top) and lateral (bottom) X-ray views for one representative trial in each subject. The axes are indicated with solid (flexion phase) and dashed lines (extension phase). The numbers next to the axes indicates the corresponding step numbers (1-4: steps in flexion; $\geqslant 5$ : steps in extension). Note that these results represent the location of the helical axes relative to the tibia. The femur is drawn for illustrative purposes in the neutral (extended) position only.

considerable changes during the stance phase, which were consistent between subjects. The FHA demonstrated an increasing medial inclination during steps $1-4$, after which the FHA suddenly returned to the transverse plane in step 5 , followed once more by an increase in medial inclination (Fig. 4D).

\section{Discussion}

The error analysis showed that the accuracy in determining the intersection point of the FHA with the tibial sagittal plane was about $8 \mathrm{~mm}$. The error in the orientation of the FHA relative to a tibial reference frame was in the order of magnitude of $3^{\circ}$. Assuming random errors, the final error in the average of five trials would be a factor of $\sqrt{ } 5$ lower, i.e. about $3.5 \mathrm{~mm}$ error in helical axis position. This error was small enough that several important aspects of the FHA could be observed during the stance phase of running in all three subjects. We were unable to estimate systematic errors in FHA, such as due to impact related movement of the pins relative to bone. However, the rather coarse temporal resolution of our analysis (four time steps for the flexion phase) is such that these high-frequency events would not affect the results. When considering the use of skin markers for a similar analysis, much larger systematic errors would be expected.

The orientation of the FHA was predominantly in a medio-lateral direction, with deviation and inclination not exceeding $25^{\circ}$ (Fig. 4C and D). A pure flexion/extension motion would occur strictly around a medio-lateral axis. Therefore, the amounts of inclination and deviation of the FHA are a measure of how much coupled rotation is occurring about the proximal-distal and anterior-posterior axes, respectively. The FHA was generally inclined on the medial side for all trials and subjects. This result is in agreement with previous in vitro studies (Blankevoort et al., 1990) and an in vivo equine study (van den Bogert, 1991). On the lateral side, the FHA was close to the tibial plateau, indicating a pure rolling motion, while on the medial side the FHA was more proximal, indicating a rotation about 
A

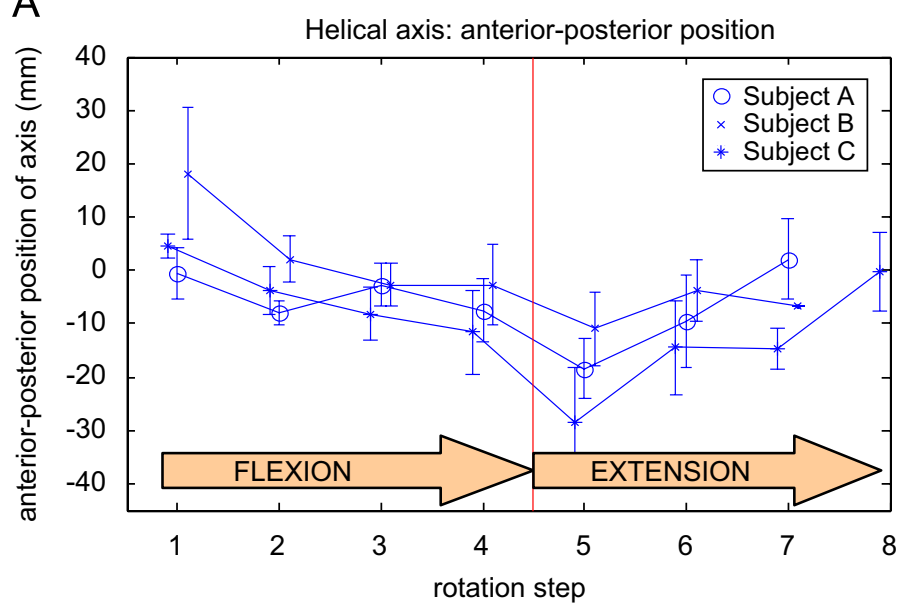

B

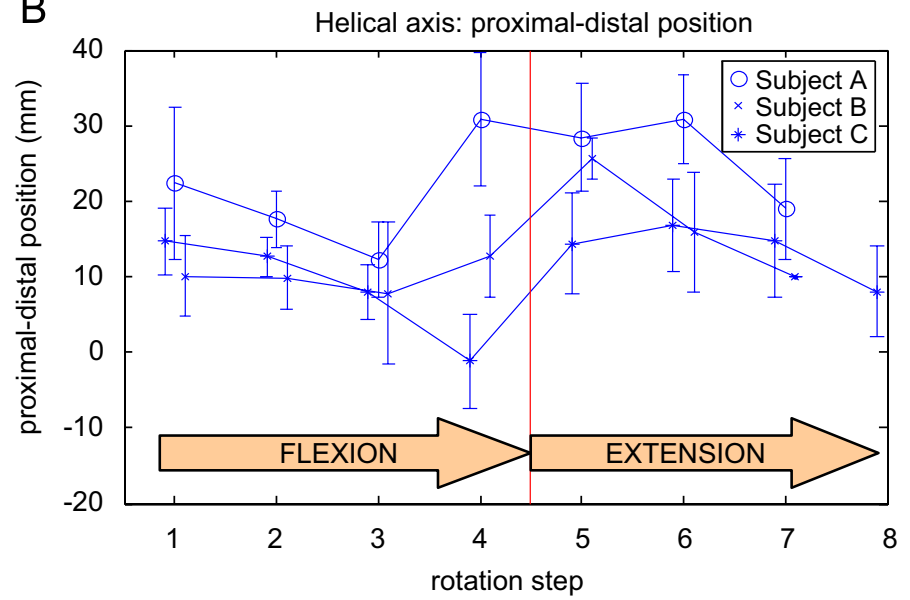

$\mathrm{C}$

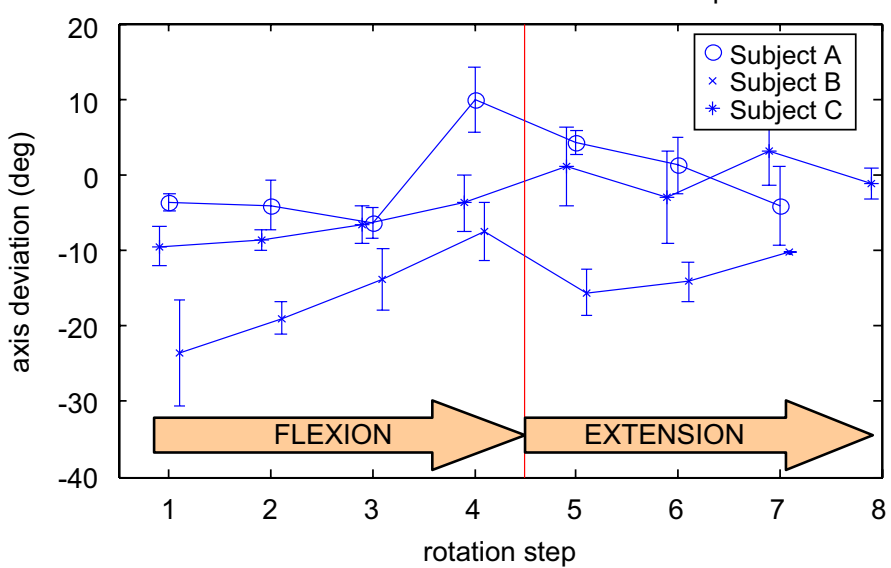

Helical axis: inclination from tibial transverse plane

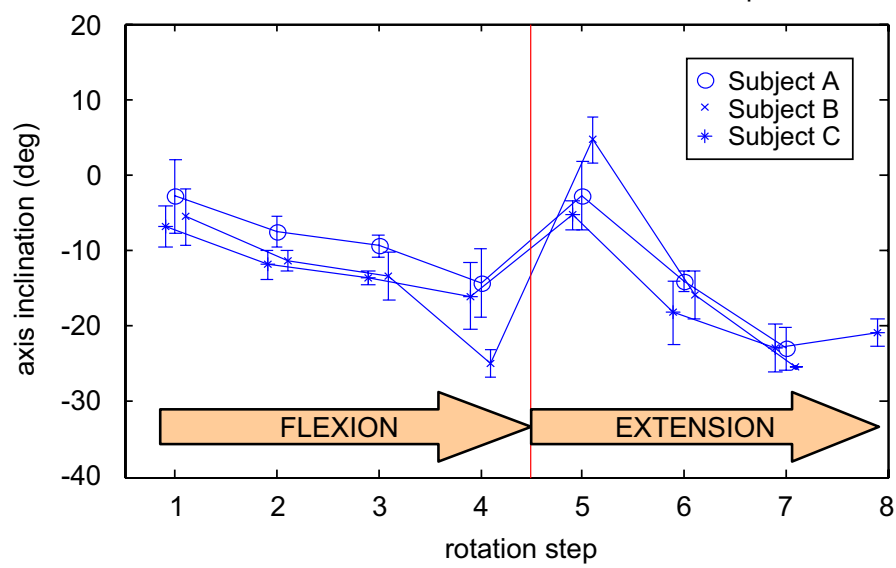

Fig. 4. . Orientation and position of the FHA during of the stance phase of running. The flexion phase in each trial was divided into exactly four rotation steps of about $6^{\circ}$, and the same step size was used for the extension phase (Fig. 2). The red line indicates the time at which the peak flexion angle ( $30-40^{\circ}$ ) occurred. The position of the FHA is expressed in the anatomical tibial coordinate system (ATCS) by the anterior-posterior (A) and proximal-distal (B) coordinates of its intersection with the tibial sagittal plane. Positive values on the vertical axes indicate anterior and proximal positions, respectively, with respect to the top of the intercondylar eminence of the tibia. The orientation is represented by the inclination (C) and deviation (D) with respect to the tibial anatomical planes. Positive values on the vertical axes correspond to an axis of which the medial end points proximally and posteriorly, respectively. Each of the three subjects is represented by the mean and standard deviation of five running trials.

the epicondyle with little rollback (Fig. 3). This is consistent with recent human in vivo studies showing a medial pivot (Komistek et al., 2003). The amount of medial inclination increased during flexion (Fig. 4D). Interestingly, during the first extension step, the FHA exhibited very little inclination in all subjects. This is consistent with our previous observation that internal tibial rotation reaches its peak just after peak flexion (Reinschmidt et al., 1997b), indicating a laxity or hysteresis in the movement coupling between flexion and internal rotation.

The point of intersection of the FHA with the tibial sagittal bisector can be regarded as the center of rotation of the knee joint in a sagittal plane. There was a tendency for the axis to move posteriorly with increasing flexion, a phenomenon typically referred to as the femoral rollback (Fig. 4A). This finding is in agreement with the classical two-dimensional four-bar linkage model (Huson, 1974) which predicts that the FHA passes through the intersec- tion of the cruciate ligaments, which moves posteriorly with increasing flexion. Similar predictions are made by 3-D mechanism models (Wilson et al., 1998). In the extension phase of running stance, the FHA moved in the opposite direction, i.e. anteriorly, with increasing extension (Fig. 4A). There was, however, about $10 \mathrm{~mm}$ of posterior shift in the FHA between motion steps 4 and 5 (Fig. 4A) which was unexpected. Since steps 4 and 5 represent the same flexion angles, such a shift is not consistent with mechanism models in which flexion is the only independent kinematic variable (Delp et al., 1990; Wilson et al., 1998). This additional shift, which occurred in all subjects, may be caused either by a passive hysteresis mechanism, or by muscle forces. The proximal-distal position of the FHA was initially about $15 \mathrm{~mm}$ from the tibial plateau and gradually moved distally (Fig. 4B), with a proximal shift of about $10 \mathrm{~mm}$ between steps 3 and 5 in all subjects. Fig. 4B can be interpreted as the ratio between 
rolling and gliding in the knee. A value of zero indicates that the FHA is on the tibial plateau, and the femur rolls without gliding. An increasing distance between FHA and tibial plateau indicates an increasing amount of gliding between the articular surfaces, for a given flexion movement.

These findings have important implications for musculoskeletal modeling. The FHA translated posteriorly by 20-30 mm during flexion, which implies a similar increase in the moment arm of the quadriceps. Consequently, large errors in inverse dynamic estimates of quadriceps force may occur if a fixed axis is assumed. It would be impractical to measure the FHA as part of a routine inverse dynamic analysis, but based on our result, a general model for FHA motion during the gait cycle, may be developed. Unfortunately, a similar analysis on our walking data was difficult because the amount of flexion during the stance phase (when muscle forces are largest) was too small for a reliable estimation of FHA.

The flexion-dependent axis position plays a role in neuromuscular control by acting as a stabilizing mechanism against buckling of the lower extremity (Wagner and Blickhan, 2003), which is important in running stance (Hardin et al., 2000). A further posterior shift of $10 \mathrm{~mm}$ occurred between steps 4 and 5, without change in flexion angle. This can be explained by the internal rotation of the tibia that occurs between steps 4 and 5 (Reinschmidt et al., 1997b), which occurs as a lateral rollback with medial pivot (Komistek et al., 2003). This phenomenon may produce additional stability against buckling, since the transition from eccentric (step 4) to concentric (step 5) state of the quadriceps function would cause muscle force to decrease due to the intrinsic force-velocity relationship of muscle. A $10 \mathrm{~mm}$ increase in moment arm would automatically compensate for this loss of force. Mechanism models with only one degree-of-freedom are unable to display such behavior. With more degrees-of-freedom in a joint model, realistic motion is possible but the axis of rotation will become dependent on the combined forces of ligaments, articular contact, and muscles. Further research is needed to elucidate the role of each of these elements in the control of the axis of rotation in the knee.

The results should also be considered in relation to the design of knee prostheses, which aim to replicate the function of the natural joint. Constrained prostheses offer improved stability and can be designed with cams to produce a helical axis that moves posteriorly as a function of flexion, to restore normal quadriceps function (Most et al., 2003). The present study, however, shows that the natural knee also has significant non-flexion-related changes in rotation axis that may be important for neuromuscular control during high-load activities, and these cannot be replicated by existing constrained mechanisms.

The methods used in this study allowed the in vivo determination of the knee joint axes during actual locomotion. The rendering of the FHA on stereo X-rays of the corresponding subject allows the display of the axes relative to the subject-specific anatomy (Fig. 3). This method for presentation is independent of coordinate systems, and allows for an unambiguous interpretation. When 3-D image data are available, rather than plane $\mathrm{X}$-rays, interactive 3-D animation should be even more effective. Such graphical techniques offer the possibility to present 3-D motion data for the knee joint without the problem of "kinematic cross-talk" that renders 3-D joint angles sensitive to small errors in the definition of anatomical coordinate axes (Blankevoort et al., 1990; Ramakrishnan and Kadaba, 1991). It should be noted, however, that quantitative comparisons between subjects or movement conditions can only be performed when the FHA position and orientation are quantified with respect to a well-defined anatomical coordinate system, as in Fig. 4.

It is concluded from this study that the axis of rotation in knee joint during the stance phase of running moves by about $30 \mathrm{~mm}$ in the sagittal plane, and undergoes changes in orientation of about $15^{\circ}$. These axis movements, when described as a function of flexion angle, had considerable hysteresis, which was consistent between subjects. These observations have important implications for the in vivo analysis of muscle function through inverse dynamic models and for design of knee prostheses and orthoses that allow muscles to function as they do in the natural joint.

\section{Conflict of interest}

None of the authors of this manuscript have a conflict of interest.

\section{Acknowledgments}

This study was supported by grants from the Whitaker Foundation (to A.J.vdB.), the Olympic Oval Endowment Fund of Calgary (to C.R.), and the Swedish Defense Material Administration (to A.L.). The contributions of Alex Stacoff, Andrzej Stano, Anna Josephson and Sandro Nigg are greatly appreciated. We also thank Stig Drevemo and Christopher Johnston of the Swedish Agricultural University in Uppsala for allowing us to borrow two cameras.

\section{References}

Bell, A.L., Pedersen, D.R., Brand, R.A., 1990. A comparison of the accuracy of several hip center location prediction methods. Journal of Biomechanics 23, 617-621.

Besier, T.F., Sturnieks, D.L., Alderson, J.A., Lloyd, D.G., 2003. Repeatability of gait data using a functional hip joint centre and a mean helical knee axis. Journal of Biomechanics 36, 1159-1168.

Blaha, J.D., Mancinelli, C.A., Simons, W.H., Kish, V.L., Thyagarajan, G., 2003. Kinematics of the human knee using an open chain cadaver model. Clinical Orthopaedics 410, 25-34. 
Blankevoort, L., Huiskes, R., de Lange, A., 1990. Helical axes of passive knee joint motions. Journal of Biomechanics 23, 1219-1229.

Delp, S.L., Loan, J.P., Hoy, M.G., Zajac, F.E., Topp, E.L., Rosen, J.M., 1990. An interactive graphics-based model of the lower extremity to study orthopaedic surgical procedures. IEEE Transactions on Biomedical Engineering 37, 757-767.

Dennis, D., Komistek, R., Scuderi, G., Argenson, J.N., Insall, J., Mahfouz, M., Aubaniac, J.M., Haas, B., 2001. In vivo threedimensional determination of kinematics for subjects with a normal knee or a unicompartmental or total knee replacement. Journal of Bone and Joint Surgery (American) 83, S104-S115.

Fantozzi, S., Benedetti, M.G., Leardini, A., Banks, S.A., Cappello, A., Assirelli, D., Catani, F., 2003. Fluoroscopic and gait analysis of the functional performance in stair ascent of two total knee replacement designs. Gait and Posture 17, 225-234.

Fleming, B.C., Brattbakk, B., Peura, G.D., Badger, G.J., Beynnon, B.D., 2002. Measurement of anterior-posterior knee laxity: a comparison of three techniques. Journal of Orthopaedic Research 20, 421-426.

Grood, E.W., Suntay, W.J., 1983. A joint coordinate system for the clinical description of three-dimensional motions: applications to the knee. Journal of Biomechanical Engineering 105, 136-144.

Hardin, E.C., Hamill, J., van den Bogert, A.J., 2000. Adaptation of running kinematics to surface and footwear. In: Twentyfourth Annual Meeting of the American Society of Biomechanics, Chicago IL, pp. 257-258.

Hill, P.F., Vedi, V., Williams, A., Iwaki, H., Pinskerova, V., Freeman, M.A.R., 2000. Tibiofemoral movement 2: the loaded and unloaded living knee studied by MRI. Journal of Bone and Joint Surgery (British) 82-B, 1196-1198.

Huson, A., 1974. Biomechanische Probleme des Kniegelenks. Orthopädie 3, 119-126.

Iwaki, H., Pinskerova, V., Freeman, M.A.R., 2000. Tibiofemoral movement 1: the shapes and relative movements of the femur and tibia in the unloaded cadaver knee. Journal of Bone and Joint Surgery (British) 82-B, 1189-1195.

Jonsson, H., Karrholm, J., 1994. Three-dimensional knee joint movements during a step-up: evaluation after anterior cruciate ligament rupture. Journal of Orthopaedic Research 12, 769-779.
Komistek, R.D., Dennis, D.A., Mahfouz, M., 2003. In vivo fluoroscopic analysis of the normal human knee. Clinical Orthopaedics and Related Research 410, 69-81.

Lafortune, M.A., Cavanagh, P.R., Sommer III, H.J., Kalenak, A., 1992. Three-dimensional kinematics of the human knee during walking. Journal of Biomechanics 25, 347-357.

Most, E., Zayontz, S., Li, G., Otterberg, E., Sabbag, K., Rubash, H.E., 2003. Femoral rollback after cruciate-retaining and stabilizing total knee arthroplasty. Clinical Orthopaedics and Related Research 410, 101-113.

Ramakrishnan, H.K., Kadaba, M.P., 1991. On the estimation of joint kinematics during gait. Journal of Biomechanics 24, 969-977.

Reinschmidt, C., van den Bogert, A.J., 1997. KineMat: A MATLAB ${ }^{\mathrm{TM}}$ toolbox for the reconstruction of spatial marker positions and for the analysis of three-dimensional joint movements. International Society of Biomechanics, 〈http://www.isbweb.org/software/movanal/kinemat $\rangle$.

Reinschmidt, C., van den Bogert, A.J., Lundberg, A., Murphy, N., Nigg, B.M., Stacoff, A., Stano, A., 1997a. Tibiofemoral and tibiocalcaneal motion during walking: skin vs. bone markers. Gait and Posture 6 , 98-109.

Reinschmidt, C., van den Bogert, A.J., Nigg, B.M., Lundberg, A., Murphy, N., 1997b. Effect of skin movement on the analysis of skeletal knee joint motion during running. Journal of Biomechanics 30, 729-732.

Söderkvist, I., Wedin, P-A., 1995. Determining the movements of the skeleton using well-configured markers. Journal of Biomechanics 26, 1473-1477.

Spoor, C.E., Veldpaus, F.E., 1980. Rigid body motion calculated from spatial co-ordinates of markers. Journal of Biomechanics 13, 391-393.

van den Bogert, A.J., 1991. Kinematical analysis of the equine knee joint. European Journal of Morphology 29, 131-132.

Wagner, H., Blickhan, R., 2003. Stabilizing function of antagonistic neuromusculoskeletal systems: an analytical investigation. Biological Cybernetics 89, 71-79.

Wilson, D.R., Feikes, J.D., O'Connor, J.J., 1998. Ligaments and articular contact guide passive knee flexion. Journal of Biomechanics 31, $1127-1136$.

Woltring, H.J., Huiskes, R., de Lange, A., Veldpaus, F.E., 1985. Finite centroid and helical axis estimation from noisy landmark measurements in the study of human joint kinematics. Journal of Biomechanics $18,379-389$. 